\title{
Intervenciones de atención farmacéutica en el control glicémico de pacientes con diabetes mellitus tipo 2
}

\author{
DOI 10.5377/alerta.v4i3.11206 \\ Karla Janet Campos Villalta \\ Facultad de Química y Farmacia, Universidad de El Salvador. \\ Correspondencia \\ $\checkmark$ karla.campos@ues.edu.sv \\ (D) $0000-0001-9177-9432$
}

\begin{abstract}
Resumen
Introducción. La diabetes mellitus tipo 2 genera un incremento de riesgo de daño, tanto a nivel microvascular como a nivel macrovascular, lo que da lugar a una disminución en la calidad de vida. En años recientes ha habido numerosos esfuerzos por implementar intervenciones de atención farmacéutica para mejorar el control y evitar la progresión de esta enfermedad. Objetivo. Conocer el efecto de las intervenciones de atención farmacéutica en el control glicémico de pacientes ambulatorios con diabetes mellitus tipo 2. Metodología. Se efectuaron búsquedas en Medline y en Cochrane Registro Central de Ensayos Controlados para obtener ensayos controlados aleatorios que evaluaran la efectividad de las intervenciones de atención farmacéutica provistas por farmacéuticos comunitarios, clínicos u hospitalarios, dirigidas a pacientes con diabetes mellitus tipo 2 a nivel ambulatorio, en comparación con la atención habitual. Resultados. En el grupo de intervención, la disminución en la diferencia media neta de hemoglobina glicosilada fue estadísticamente significativa en 27 estudios, pues el rango osciló entre 0.4 \% y $3.3 \%$; en 14 estudios, dicha disminución fue estadísticamente significativa y superior o igual a $1 \%$. En cuanto a la disminución en la diferencia media neta mostrada en el cambio de hemoglobina glicosilada, desde el inicio hasta el final del seguimiento, entre el grupo de intervención y el grupo control, fue significativa en 22 estudios y el rango osciló entre 0.3 y $2.3 \%$. Conclusión. Las evidencias recopiladas demuestran el efecto significativo que tienen las intervenciones de atención farmacéutica en la mejora del control glicémico de pacientes ambulatorios con diabetes mellitus tipo 2.
\end{abstract}

Palabras clave

Servicios farmacéuticos, diabetes mellitus tipo 2, hemoglobina A glucada.

\begin{abstract}
Introduction. Type 2 diabetes mellitus generates a risk increase of damage both at the microvascular level and at the macrovascular level, which leads to a decrease in quality of life. In recent years, there have been many efforts to implement pharmaceutical care interventions in order to improve the control and to avoid progression of this disease. Objective. To know the effect of pharmaceutical care interventions on glycemic control of type 2 diabetes mellitus outpatients. Methodology. It was searched in Medline and Cochrane Central Register of Controlled Trials to obtain Randomized Controlled Trials evaluating the effectiveness of Pharmaceutical Care interventions provided by community, clinical or hospital pharmacists, aimed to type 2 diabetes mellitus outpatients in comparison with usual care. Results. In the intervention group, the decrease in the net mean difference of glycosylated hemoglobin was statistically significant in 27 studies, the range was between: $0,4 \%$ and $3,3 \%$; in 14 studies this decrease was statistically significant and greater than or equal to $1 \%$. Regarding the decrease in the net mean difference shown in the change in glycosylated hemoglobin, from the beginning to the end of the follow-up, between the intervention group and the control group, it was significant in 22 studies and the range was between: 0,3 and 2,3\%. Conclusion. The evidence collected demonstrates the significant effect that pharmaceutical care interventions have in the improvement of glycemic control in type 2 diabetes mellitus outpatients.
\end{abstract}

Keywords

Pharmaceutical services, diabetes mellitus type 2, glycated hemoglobin A.

\section{G}

ACCESO ABIERTO

Pharmaceutical care interventions in the glycemic control of patients with type 2 diabetes mellitus

Citación recomendada: Campos Villalta KJ.

Intervenciones de atención farmacéutica en el control glicémico de pacientes con diabetes mellitus tipo 2 . Alerta. 2021; 4(3):159-169. DOI: 10.5377/alerta.v4i3.11206

Recibido:

21 de marzo de 2021

Aceptado:

10 de junio de 2021

Publicado:

26 de julio de 2021

\section{Contribución de autoría:} La autora realizó todo el proceso de revisión sistemática de los artículos originales retomados en la revisión.

\section{Conflicto de intereses:}

Se declara no tener ningún tipo de conflicto de interés. 


\section{Introducción}

A escala mundial se calcula que 422 millones de adultos tenían diabetes mellitus (DM) en 2014, en comparación con 108 millones en 1980. Desde 1980, la prevalencia mundial de la DM ha ascendido a casi el doble (del 4,7 $\%$ al 8,5 \%) en la población adulta. Esto corresponde con un aumento de sus factores de riesgo, tales como el sobrepeso y la obesi$\mathrm{dad}^{1}$. A pesar de las terapias efectivas disponibles para ayudar a los pacientes a controlar su DM, la evidencia demuestra que el logro de los objetivos recomendados sigue siendo subóptimo para estos pacientes². Las complicaciones relacionadas con la DM, macroangiopáticas y microangiopáticas incluidas la nefropatía, la retinopatía, la neuropatía, los accidentes cerebrovasculares y las enfermedades cardiovasculares, están aumentando en paralelo con las altas tasas de DM no controlada o produciéndose en corto tiempo ${ }^{3}$. La hemoglobina glicosilada (HbA1c) refleja los niveles de glucosa en sangre durante los últimos 120 días y es el biomarcador de referencia para la evaluación del control de la DM y la predicción de complicaciones graves ${ }^{4}$.

El manejo de la DM no solo se limita a los medicamentos, sino que requiere un enfoque multidimensional, que incluye aspectos relacionados con el estilo de vida (dieta, ejercicio físico, autocontrol) y seguimiento continuo ${ }^{3}$, así como, educación diabetológica para aprender a conocer la enfermedad y a vivir con ella. De acuerdo con la Asociación Americana de Diabetes, el óptimo manejo de la DM requiere un enfoque organizado, sistemático y la participación de un equipo coordinado de profesionales dedicados a la atención sanitaria que trabajen en un entorno donde la atención de alta calidad centrada en el paciente sea una prioridad y a la vez recomienda la incorporación del farmacéutico al equipo de atención ${ }^{5}$. En este contexto, los farmacéuticos también pueden contribuir positivamente al manejo de la DM al proporcionar programas de atención farmacéutica, que implican trabajar estrechamente con el paciente y otros profesionales de la salud en el diseño, implementación y monitoreo de planes terapéuticos para lograr resultados específicos que mejoren la calidad de vida del paciente 6 . Se trata de una práctica profesional en la que el farmacéutico se responsabiliza de las necesidades del paciente relacionadas con los medicamentos.

En una revisión sistemática publicada por Pousinho et alo (2016), en la cual se evaluaron los efectos de varias intervenciones farmacéuticas en el tratamiento de la DM tipo 2, realizadas en varios países y en diferentes centros de atención sanitaria, como farmacias comunitarias, clínicas de atención primaria y hospitales, la HbA1c se evaluó en 26 estudios, de los cuales 24 reportaron una mayor reducción en este resultado en el grupo de intervención en comparación con el grupo de control, con una diferencia en el cambio entre los grupos que va desde 0,18\% a 2,1\%. Incluso hay que destacar que en 11 estudios la reducción de HbA1c en el grupo de intervención fue mayor que la registrada en el grupo control en aproximadamente 1 \% o más.

En las últimas dos décadas, ha habido un creciente cuerpo de bibliografía que evalúa la eficacia de la participación de los farmacéuticos en el tratamiento de los pacientes diabéticos en diversos entornos; sin embargo, solo se han publicado unas pocas revisiones sistemáticas sobre este tema y algunas de ellas evaluaron las intervenciones de los farmacéuticos en pacientes con DM tipo 1 y con DM tipo 2. Otras revisiones incluyeron otros tipos de estudios (por ejemplo, estudios de cohortes), además de los ensayos controlados aleatorios. Algunas revisiones, por su parte, se enfocaron en un ámbito sanitario en particular, ya sea en farmacia comunitaria, clínica u hospitalaria.

El propósito de esta revisión sistemática es brindar una visión global de la efectividad de las intervenciones farmacéuticas en el manejo de la DM tipo 2 específicamente, enfocándose en los resultados clínicos e incluyendo solo los estudios más robustos, es decir, ensayos controlados aleatorios, sin imponer restricciones en cuanto al ámbito sanitario y año de publicación del estudio.

El objetivo de la presente revisión sistemática es conocer el efecto de las intervenciones de atención farmacéutica en el control glicémico de pacientes ambulatorios con DM tipo 2. El enfoque PICOS se utilizó para establecer la siguiente pregunta de investigación: En pacientes con DM tipo 2 con HbA1c > 7\%, ¿las intervenciones de atención farmacéutica son más eficaces que la atención habitual para controlar el problema de salud?

Siguiendo las pautas de la guía PRISMA, se consideró a todos los pacientes con DM tipo 2 que tuvieran una HbA1c mayor que $7 \%$, como la población/problema; las intervenciones de atención farmacéutica provistas por farmacéuticos se establecieron como la intervención; la atención habitual provista por médicos, enfermera y/o farmacéuticos fue la comparación; el cambio medio de los niveles de HbA1c del grupo de intervención y control fueron los resultados esperados. Se seleccionaron los ensayos controlados aleatorios (ECA) como el diseño de los estudios a seleccionar. 


\section{Metodología}

\section{Fuente de datos y estrategia de búsqueda}

Una revisión sistemática de la literatura contenida en las bases de datos Medline (vía PubMed) y Registro Cochrane Central de Ensayos Controlados (CENTRAL) fue llevada a cabo en enero de 2021, realizando búsquedas sin restricciones en cuanto al año de publicación de los estudios, para identificar el efecto de las intervenciones de atención farmacéutica en el control glicémico de pacientes ambulatorios con DM tipo 2. La declaración PRISMA, para informar revisiones sistemáticas y metaanálisis de estudios que evalúan intervenciones sanitarias ${ }^{7}$, fue tomada en cuenta para realizar la presente revisión. El protocolo no fue registrado en una base de datos internacional de revisiones sistemáticas.

La estrategia de búsqueda usada en Medline (vía PubMed) es la siguiente:

(diabetes mellitus, type 2[MeSH Terms]) AND («(c(()(community pharmacy services [MeSH Terms]) OR (medication therapy management[MeSH Terms])) OR (pharmaceutical care[MeSHTerms])) OR (clinical pharmacy services[MeSH Terms])) OR (clinical pharmacist[MeSH Terms])) OR (community pharmacies[MeSH Terms])) OR («pharmacist intervention»)) OR («medication review»)) Filtros: Randomized Controlled Trial.
En cuanto a la estrategia de búsqueda usada en Registro Cochrane Central de Ensayos Controlados (CENTRAL) se describe a continuación: \#1 «community pharmacy services» / \#2 «medication therapy management» / \#3 «Pharmaceutical Services» / \#4 «Pharmacy Service, Hospital» / \#5 «pharmacist intervention» / \#6 \#1 OR \#2 OR \#3 OR \#4 OR\#5 / \#7 «Diabetes Mellitus, Type 2»/\#8 \#7 AND \#6.

\section{Criterios de inclusión y exclusión de los estudios}

Los criterios de inclusión fueron los siguientes: 1) ensayo controlado aleatorio (ECA); 2) pacientes ambulatorios con diagnóstico confirmado de DM tipo 2; 3) intervenciones de atención farmacéutica provistas por farmacéuticos comunitarios, clínicos u hospitalarios, incluyendo aquellas realizadas dentro de un equipo multidisciplinario de salud. 4) Cada artículo debe reportar los niveles de $\mathrm{HbA1c}$ al inicio y al final del estudio o el cambio medio en estos valores, tanto del grupo control como del grupo de intervención. 5) Sin restricciones en cuanto al año de publicación del estudio.

Los criterios de exclusión fueron los siguientes: 1) los artículos que no estén en idioma inglés o en español, 2) las intervenciones de atención farmacéutica enfocadas principalmente en la adherencia de la medicación.

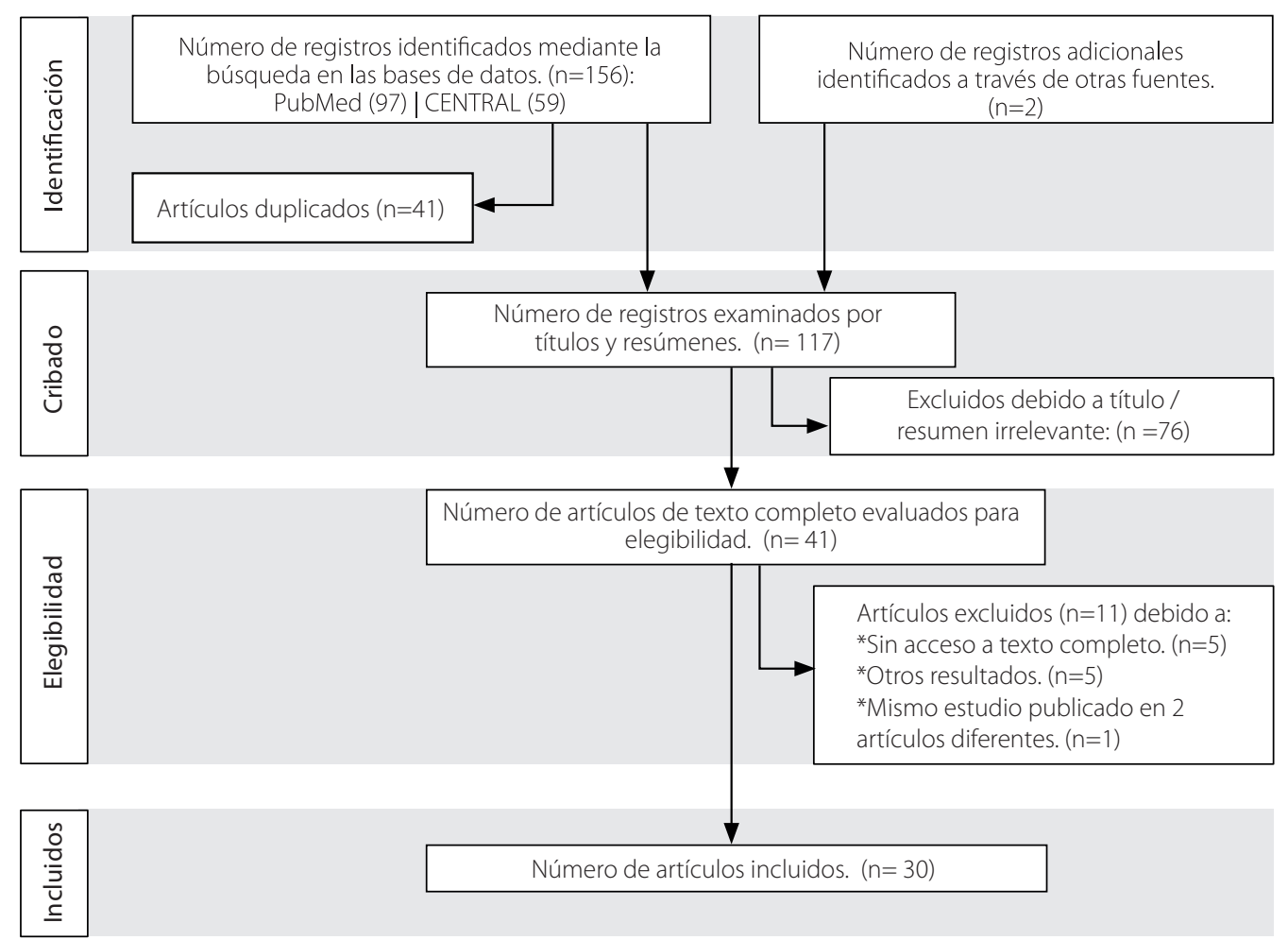

Figura 1. Diagrama de Flujo del proceso de selección de los estudios para la revisión sistemática, basado en los "Elementos de Informe Preferidos para revisiones sistemáticas y metaanálisis" (PRISMA). 


\section{Selección de estudios}

Todo el proceso de selección de los estudios, desde la identificación, el cribado, la elegibilidad y la inclusión fue llevado a cabo por la autora del artículo y es presentado en el diagrama de flujo PRISMA, en la Figura 1.

\section{Extracción de datos}

La siguiente información fue extraída de los 30 artículos de texto completo, incluidos en la presente revisión: apellido del primer autor, año de publicación, país y ámbito del estudio, duración del período de seguimiento, número de pacientes del grupo de intervención y control, breve descripción de las intervenciones farmacéuticas realizadas, tipo de entrega de la intervención farmacéutica, tipo de citas, encargado de la atención habitual, resultados clínicos y humanísticos medidos, valores de HbA1c inicial y final tanto del grupo de intervención como del grupo control. Esta información fue vaciada en una matriz diseñada en el programa Microsoft Excel.

\section{Evaluación del riesgo de sesgo}

Todos los estudios incluidos se evaluaron de acuerdo con la herramienta Cochrane de riesgo de sesgo, que toma en consideración los siguientes criterios: 1) generación de secuencia aleatoria; 2) ocultación de la asignación; 3) cegamiento de los participantes y del personal; 4) cegamiento de los evaluadores de los resultados; 5) datos de resultados incompletos; 6) notificación selectiva de los resultados y 7) otras fuentes de sesgo. Se verificó cada uno de los criterios para cada estudio, clasificando el riesgo en bajo, alto o poco claro, según correspondiera de acuerdo con lo explicado en detalle en el Manual Cochrane de revisiones sistemáticas de intervenciones ${ }^{8}$.

\section{Resultados}

\section{Flujo PRISMA para la selección de estudios}

La búsqueda inicial identificó 156 estudios, 97 se encontraban en PubMed y 59 en CENTRAL, de los cuales 41 estaban duplicados. Adicional a lo anterior, 2 fueron identificados a través de otras fuentes. Durante el cribado, 117 estudios fueron examinados por títulos y/o resúmenes, de los cuales fueron excluidos 76, por ser irrelevantes para la pregunta de investigación. Los 41 estudios restantes se sometieron a una evaluación de texto completo utilizando los criterios de inclusión y exclusión establecidos anteriormente; durante esta etapa se excluyeron 11 estudios, debido a los siguientes aspectos: en 5 estudios no se tuvo acceso al texto completo, otros 5 presentaban otro tipo de resultados que no eran de interés para la presente revisión y un mismo estudio fue publicado en dos artículos diferentes. Cabe destacar que esto se identificó yuxtaponiendo autores, tamaño de muestra y resultados. Por lo tanto, un total de 30 estudios se incluyeron en la presente revisión cualitativa ${ }^{9-38}$.

\section{Riesgo de sesgo}

La Figura 2 muestra el porcentaje de presentación del riesgo de sesgos para cada uno de los estudios incluidos en la presente revisión sistemática; 3 criterios en particular presentaron en su mayoría un riesgo bajo: generación de secuencia aleatoria (66\%); datos de resultados incompletos $(86,66 \%)$ y notificación selectiva de los resultados (90\%). Existen 4 criterios, en su mayoría con un riesgo poco claro: ocultación de la asignación (63,33\%); cegamien-

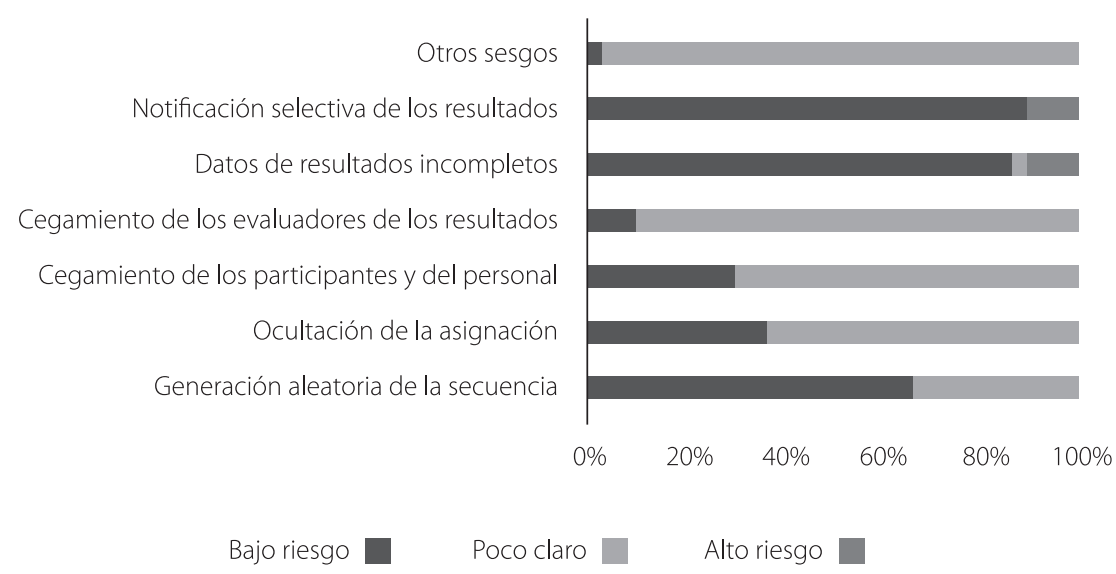

Figura 2. Porcentaje de presentación del riesgo de sesgo de los ECA incluidos en la revisión. 
to de los participantes y del personal 70 $\%$; cegamiento de los evaluadores de los resultados $90 \%$ y otras fuentes de sesgo $96 \%$, debido básicamente a la limitada información proporcionada al respecto en los diferentes estudios.

\section{Características de los \\ estudios incluidos en la revisión sistemática}

De los 30 artículos, 11 estudios fueron realizados en el continente americano (EE. UU [n=9] y Brasil [n=2]); 5 en Europa (Bélgica, España, Francia, Chipre y Reino Unido); 12 en Asia (Irán $[n=3]$, Jordania [ $n=2]$, China, Emiratos Árabes
Unidos, Malasia, Pakistán, Singapur, Tailandia y Taiwán) y 2 en Oceanía (Australia). En general, los 30 estudios incluyeron un total de 2796 personas en el grupo de intervención (Gl) y 2852 personas en el grupo control (GC), haciendo un total de 5648 participantes. Todos los ensayos fueron realizados con pacientes ambulatorios, en los siguientes ámbitos sanitarios: 10 en clínicas de atención primaria, 9 en farmacias comunitarias, 8 en hospitales, 1 en clínica de atención secundaria y 2 en ambientes no especificados. La duración del seguimiento a los pacientes fue estratificada de la siguiente manera: $<6$ meses $(n=6)$, entre 6 y 12 meses $(n=22)$ y $>12$ meses $(n=2)$. Esta información se detalla en la Tabla 1.

Tabla 1. Principales Características de los Ensayos Controlados Aleatorios incluidos en la Revisión Sistemática.

\begin{tabular}{|c|c|c|c|c|c|c|c|c|c|c|c|c|c|}
\hline $\mathrm{N}^{\circ}$ & $\begin{array}{l}\text { Apellido } 1^{\mathrm{er}} \text { autor } \\
\text { y año publicación }\end{array}$ & País & ámbito de estudio & $\begin{array}{l}\text { Tiempo de } \\
\text { seguimiento } \\
\text { (meses) }\end{array}$ & $\mathrm{Gl}$ & GC & $\mathrm{N}^{\circ}$ & $\begin{array}{l}\text { Apellido } 1^{\text {er }} \text { autor y } \\
\text { año publicación }\end{array}$ & País & $\begin{array}{l}\text { ámbito de } \\
\text { estudio }\end{array}$ & $\begin{array}{l}\text { Tiempo de } \\
\text { seguimiento } \\
\text { (meses) }\end{array}$ & $\mathrm{Gl}$ & $\mathrm{GC}$ \\
\hline 2 & Ali, et al.., $2012^{10}$ & $\begin{array}{l}\text { Reino } \\
\text { Unido }\end{array}$ & $\begin{array}{c}\text { Farmacia } \\
\text { Comunitaria }\end{array}$ & 12 & 23 & 23 & 17 & Krass, et al., $2007^{25}$ & Australia & $\begin{array}{c}\text { Farmacia } \\
\text { Comunitaria }\end{array}$ & 6 & 149 & 140 \\
\hline 3 & $\begin{array}{l}\text { Ayadurai, et al., } \\
\qquad 2018^{11}\end{array}$ & Malasia & $\begin{array}{c}\text { Clínica de atención } \\
\text { primaria }\end{array}$ & 6 & 49 & 63 & 18 & $\begin{array}{l}\text { Lauffenburger, et } \\
\text { al., } 2019^{26}\end{array}$ & $\begin{array}{l}\text { Estados } \\
\text { Unidos }\end{array}$ & No especificado & 12 & 678 & 684 \\
\hline 4 & $\begin{array}{l}\text { Castejón, et al., } \\
2013^{12}\end{array}$ & $\begin{array}{l}\text { Estados } \\
\text { Unidos }\end{array}$ & $\begin{array}{c}\text { Farmacia } \\
\text { Comunitaria }\end{array}$ & 3 & 19 & 24 & 19 & $\begin{array}{l}\text { Mazroui, et al., } \\
2009^{27}\end{array}$ & $\begin{array}{l}\text { Emiratos } \\
\text { Árabes } \\
\text { Unidos }\end{array}$ & Hospital & 12 & 117 & 117 \\
\hline 5 & Chan, et al., $2012^{13}$ & China & Hospital & 9 & 51 & 54 & 20 & $\begin{array}{l}\text { Mehuys, et al., } \\
2011^{28}\end{array}$ & Bélgica & $\begin{array}{l}\text { Farmacia } \\
\text { Comunitaria }\end{array}$ & 6 & 153 & 135 \\
\hline 6 & Chen, et al., $2015^{14}$ & Taiwán & $\begin{array}{c}\text { Farmacia } \\
\text { Comunitaria }\end{array}$ & 6 & 50 & 50 & 21 & $\begin{array}{l}\text { Michiels, et al., } \\
2019^{29}\end{array}$ & Francia & $\begin{array}{c}\text { Farmacia } \\
\text { Comunitaria }\end{array}$ & 12 & 160 & 162 \\
\hline 7 & $\begin{array}{l}\text { Clifford, et al., } \\
2005^{15}\end{array}$ & Australia & No especificado & 12 & 92 & 88 & 22 & $\begin{array}{l}\text { Mourao, et al., } \\
2012^{30}\end{array}$ & Brasil & $\begin{array}{l}\text { Clínica de } \\
\text { atención } \\
\text { primaria }\end{array}$ & 6 & 50 & 50 \\
\hline 8 & $\begin{array}{l}\text { Cohen, et al., } \\
2011^{16}\end{array}$ & $\begin{array}{l}\text { Estados } \\
\text { Unidos }\end{array}$ & $\begin{array}{c}\text { Clínica de atención } \\
\text { primaria }\end{array}$ & 6 & 50 & 49 & 23 & $\begin{array}{l}\text { Sarayani, et al., } \\
\qquad 2018^{31}\end{array}$ & Irán & $\begin{array}{c}\text { Farmacia } \\
\text { Comunitaria }\end{array}$ & 3 & 50 & 50 \\
\hline 9 & $\begin{array}{l}\text { Farsaei, et al., } \\
2011^{17}\end{array}$ & Irán & $\begin{array}{c}\text { Clínica de atención } \\
\text { primaria }\end{array}$ & 3 & 87 & 87 & 24 & Scott, et al., $2006^{32}$ & $\begin{array}{l}\text { Estados } \\
\text { Unidos }\end{array}$ & $\begin{array}{l}\text { Clínica de } \\
\text { atención } \\
\text { primaria }\end{array}$ & 9 & 64 & 67 \\
\hline 10 & $\begin{array}{l}\text { Fornos, et al., } \\
2006^{18}\end{array}$ & España & $\begin{array}{c}\text { Farmacia } \\
\text { Comunitaria }\end{array}$ & 13 & 56 & 56 & 25 & Siaw, et al., $2017^{33}$ & Singapur & $\begin{array}{l}\text { Clínica de } \\
\text { atención } \\
\text { primaria }\end{array}$ & 6 & 141 & 189 \\
\hline 12 & $\begin{array}{l}\text { Jacobs, et al., } \\
2012^{20}\end{array}$ & $\begin{array}{l}\text { Estados } \\
\text { Unidos }\end{array}$ & $\begin{array}{c}\text { Clínica de atención } \\
\text { primaria }\end{array}$ & 12 & 72 & 92 & 27 & Taveira, et al., $2011^{35}$ & $\begin{array}{l}\text { Estados } \\
\text { Unidos }\end{array}$ & $\begin{array}{l}\text { Clínica de } \\
\text { atención } \\
\text { primaria }\end{array}$ & 6 & 44 & 44 \\
\hline 13 & $\begin{array}{c}\text { Jahangard- } \\
\text { Rafsanjani, et al., } \\
2015^{21}\end{array}$ & Irán & $\begin{array}{c}\text { Farmacia } \\
\text { Comunitaria }\end{array}$ & 5 & 45 & 40 & 28 & $\begin{array}{l}\text { Wishah, et al., } \\
\qquad 2014^{36}\end{array}$ & Jordania & Hospital & 6 & 50 & 51 \\
\hline 14 & Jarab, et al., $2012^{22}$ & Jordania & Hospital & 6 & 77 & 79 & 29 & $\begin{array}{l}\text { Withidpanyawong, } \\
\text { et al., } 2018^{37}\end{array}$ & Tailandia & Hospital & 9 & 88 & 92 \\
\hline 15 & $\begin{array}{l}\text { Javaid, et al., } \\
2019^{23}\end{array}$ & Pakistán & $\begin{array}{c}\text { Clínica de atención } \\
\text { primaria }\end{array}$ & 9 & 83 & 52 & 30 & Wu, et al., $2018^{38}$ & $\begin{array}{l}\text { Estados } \\
\text { Unidos }\end{array}$ & Hospital & 13 & 112 & 127 \\
\hline
\end{tabular}


Tabla 2. Frecuencia de implementación de los tipos de intervenciones de atención farmacéutica desarrollados en los grupos de intervención de los ensayos controlados aleatorios incluidos en la revisión sistemática.

\begin{tabular}{ll}
\hline Tipos de intervenciones de atención farmacéutica & Frecuencia de ECA que las incluyen \\
\hline 1. Intervenir sobre la educación del paciente: & -- \\
1.1 Educar sobre la enfermedad y complicaciones. & 25 \\
1.2 Educar sobre el desarrollo de habilidades: & -- \\
1.2.1 Administración y uso correcto de la medicación. & 20 \\
1.2.2 Automonitoreo de glucosa. & 19 \\
1.3 Modificar comportamientos: & -- \\
1.3.1 Autocuidado. & 20 \\
1.3.2 Adherencia a la medicación. & 22 \\
1.4 Educar sobre el conocimiento de la medicación. & 14 \\
1.5 Educar sobre el estilo de vida: & --- \\
1.5.1 Dieta. & 29 \\
1.5.2 Ejercicio. & 27 \\
1.5.3 Cesación tabáquica. & 11 \\
1.6 Educar sobre la identificación de eventos hipo e hiperglucémicos. & 11 \\
1.7 Educar sobre la identificación de efectos adversos. & 11 \\
2. Identificar y resolver Problemas Relacionados a los Medicamentos. & 11 \\
3. Realizar cambios en el tratamiento farmacológico. & -- \\
3.1 Intervenir sobre la estrategia farmacológica. (añadir, retirar o sus- \\
tituir medicamentos). \\
3.2 Intervenir sobre la dosificación de la medicación. \\
4. Derivar a otros profesionales de salud. & 20 \\
\hline
\end{tabular}

Por otra parte, hay que destacar que 7 estudios eran multicéntricos ${ }^{11,18,25,28-30,38 ;}$; en 2 estudios los pacientes recibieron remuneración por su participación en la investigación ${ }^{12,32}$ y de igual forma, en 2 estudios el farmacéutico recibió remuneración por su participación ${ }^{14,25}$

En cuanto a la medición de otros resultados clínicos, aparte de la $\mathrm{HbA} 1 \mathrm{c}$, en los diferentes ensayos controlados aleatorios comprendidos en la presente revisión sistemática se detalla a continuación el tipo de resultado y el porcentaje de medición en los 30 estudios: presión arterial sistólica y diastólica 83,33 \%, índice de masa corporal 76,67 \%, lipoproteínas de baja densidad 76,67 \%, glucosa capilar 66,67\%, lipoproteínas de alta densidad 63,33\%, triglicéridos $50 \%$ y por último, la presentación de eventos hipo e hiperglucémicos y visitas a emergencias y hospitalizaciones se cuantificó en el $10 \%$ de los estudios. Algunos estudios tomaron en consideración también la medición de resultados humanísticos. Se detalla a continuación el tipo de resultado y el porcentaje de medición en los 30 estudios de la siguiente manera: adherencia a la medicación 43,33 \%, implementación de actividades de autocuidado $30 \%$, calidad de vida 16,67\%, conocimiento de la DM 16,67\%, conocimiento de la medicación 6,67 \% y estado de salud 6,67\%.

\section{Intervenciones de atención farmacéutica}

En los ensayos controlados aleatorios incluidos en la presente revisión sistemática se implementó en los grupos de intervención una amplia variedad de intervenciones de atención farmacéutica desarrolladas por farmacéuticos, enfocadas en la educación del paciente, en la identificación y en la resolución de problemas relacionados a los medicamentos, la modificación de la estrategia farmacológica y/o la dosificación de la medicación e incluso en la derivación a otros profesionales de la salud para el abordaje de otros aspectos. En la Tabla 2 se resume la frecuencia de implementación de cada tipo de intervención de atención farmacéutica, en los grupos de intervención de los ECA incluidos en la presente revisión. Es importante destacar que 10 estudios se enfocaron principalmente en la educación del paciente $\mathrm{e}^{10,12,13,17,21,31-33,37,38}$. En los otros 20 estudios, además de realizar actividades educativas, el farmacéutico realizó cambios en la medicación del paciente, en coordinación con el médico tratante, ya sea relacionados con la estrategia farmacoterapéutica para tratar la DM tipo 2 (añadir, retirar o sustituir un medicamento), como relacionados con la dosificación de la medicación. 
Tabla 3. Valores de HbA1c del grupo de intervención y del grupo control de los ECA incluidos en la revisión sistemática.

\begin{tabular}{|c|c|c|c|c|c|c|c|c|c|c|c|}
\hline \multirow[b]{2}{*}{$\mathrm{N}^{\circ}$} & \multirow[b]{2}{*}{$\begin{array}{l}\text { Identificación del } \\
\text { estudio }\end{array}$} & \multicolumn{4}{|c|}{ HbA1c Grupo Intervención } & \multicolumn{4}{|c|}{ HbA1c Grupo Control } & \multirow[b]{2}{*}{$\begin{array}{l}\text { Diferencia } \\
\text { neta entre } \\
\text { grupos }\end{array}$} & \multirow[b]{2}{*}{$\begin{array}{c}\text { p entre } \\
\text { grupos al } \\
\text { final }\end{array}$} \\
\hline & & $\begin{array}{c}\text { Media } \\
\text { medición } \\
\text { base } \\
\left(\mathrm{DE}^{*}\right)\end{array}$ & $\begin{array}{l}\text { Media } \\
\text { medición final } \\
\text { (DE) }\end{array}$ & $\begin{array}{c}\text { Diferencia } \\
\text { media } \\
\text { neta }\end{array}$ & $\mathrm{p}$ & $\begin{array}{l}\text { Media } \\
\text { medición } \\
\text { base } \\
\text { (DE) }\end{array}$ & $\begin{array}{l}\text { Media } \\
\text { medición } \\
\text { final (DE) }\end{array}$ & $\begin{array}{c}\text { Diferencia } \\
\text { media } \\
\text { neta }\end{array}$ & $\mathrm{p}$ & & \\
\hline 1 & Aguiar, et al., $2016^{9}$ & $9,03(1,3)$ & $8,24(0,9)$ & 0,79 & $<0,001$ & $8,87(1,4)$ & $8,71(2,0)$ & 0,16 & $>0,05$ & 0,63 & 0,01 \\
\hline 2 & Ali, et al., $2012^{10}$ & $8,2(1,65)$ & $6,6(0,59)$ & 1,60 & $<0,001$ & $8,1(0,97)$ & $7,5(0,64)$ & 0,60 & 0,03 & 1,00 & $<0,001$ \\
\hline 3 & $\begin{array}{c}\text { Ayadurai, et al., } \\
2018^{11}\end{array}$ & $10,68(2,2)$ & $9,08(2,2)$ & 1,60 & $<0,001$ & $10,32(1,5)$ & $10,07(1,8)$ & 0,25 & $>0,05$ & 1,35 & $<0,001$ \\
\hline 4 & $\begin{array}{c}\text { Castejón, et al., } \\
2013^{12}\end{array}$ & $8,3(0,4)$ & $7,3(0,3)$ & 1,00 & $<0,05$ & $8,2(0,4)$ & $8,0(0,2)$ & 0,20 & $>0,05$ & 0,80 & $>0,05$ \\
\hline 5 & Chan, et al., $2012^{13}$ & $9,7(1,4)$ & $8,13(1,5)$ & 1,57 & $<0,01$ & $9,5(1,8)$ & $9,1(1,19)$ & 0,40 & $>0,05$ & 1,17 & $<0,001$ \\
\hline 7 & Clifford, et al., 2005 & $7,5\left(\mathrm{NE}^{* *}\right)$ & $7,0(\mathrm{NE})$ & 0,50 & $<0,05$ & 7,1 (NE) & $7,1(\mathrm{NE})$ & 0,00 & $<0,05$ & 0,50 & 0,002 \\
\hline 8 & Cohen, et al., $2011^{16}$ & $7,8(1,0)$ & $7,39(\mathrm{NE})$ & 0,41 & $<0,05$ & $8,1(1,4)$ & 7,9 (NE) & 0,20 & $>0,05$ & 0,21 & $>0,05$ \\
\hline 9 & Farsaei, et al., $2011^{17}$ & $9,3(1,7)$ & $7,5(1,6)$ & 1,80 & $<0,001$ & $8,9(1,1)$ & $9,0(1,2)$ & $-0,10$ & 0,317 & 1,90 & $>0,05$ \\
\hline 10 & Fornos, et al., $2006^{18}$ & $8,4(1,8)$ & $7,9(1,7)$ & 0,50 & $<0,001$ & $7,8(1,7)$ & $8,5(1,9)$ & $-0,70$ & $<0,001$ & 1,20 & $<0,001$ \\
\hline 11 & Jaber, et al., $1996^{19}$ & $11,5(2,9)$ & $9,2(2,1)$ & 2,30 & $<0,05$ & $12,2(3,5)$ & $12,1(3,7)$ & 0,10 & $>0,05$ & 2,20 & $<0,05$ \\
\hline 12 & Jacobs $2012^{20}$ & $9,5(1,1)$ & $7,7(1,3)$ & 1,80 & $<0,05$ & $9,2(1,0)$ & $8,4(1,6)$ & 0,80 & $<0,05$ & 1,00 & 0,003 \\
\hline 13 & $\begin{array}{l}\text { Jahangard-Rafsanjani } \\
\qquad 2015^{21}\end{array}$ & $7,6(1,6)$ & $6,6(1,5)$ & 1,00 & 0,0001 & $7,5(1,9)$ & $7,0(1,7)$ & 0,50 & 0,03 & 0,50 & 0,09 \\
\hline 14 & Jarab, et al., $2012^{22}$ & 8,5 (NE) & 7,7 (NE) & 0,80 & $<0,05$ & 8,4 (NE) & 8,5 (NE) & $-0,10$ & $>0,05$ & 0,90 & 0,019 \\
\hline 16 & $\begin{array}{c}\text { Korcegez, et al., } \\
2017^{24}\end{array}$ & $8,29(0,89)$ & $7,55(0,57)$ & 0,74 & $<0,001$ & $8,31(0,84)$ & $8,26(0,74)$ & 0,05 & 0,671 & 0,69 & $<0,001$ \\
\hline 17 & Krass, et al., $2007^{25}$ & $8,9(1,4)$ & $7,9(1,2)$ & 1,00 & $<0,05$ & $8,3(1,3)$ & $8,0(1,2)$ & 0,30 & $>0,05$ & 0,70 & $<0,01$ \\
\hline 18 & $\begin{array}{l}\text { Lauffenburger, et al., } \\
\qquad 2019^{26}\end{array}$ & $9,3(1,6)$ & $8,55(1,96)$ & 0,75 & $>0,05$ & $9,4(1,6)$ & $8,61(2,01)$ & 0,79 & $>0,05$ & $-0,04$ & $>0,05$ \\
\hline 19 & Mazroui, et al., $2009^{27}$ & 8,5 (NE) & 6,9 (NE) & 1,60 & $<0,001$ & 8,4 (NE) & 8,3 (NE) & 0,10 & $>0,05$ & 1,50 & $<0,05$ \\
\hline 20 & Mehuys, et al., $2011^{28}$ & $7,7(1,7)$ & $7,1(1,1)$ & 0,60 & $<0,001$ & $7,3(1,2)$ & $7,2(1,0)$ & 0,10 & 0,162 & 0,50 & 0,009 \\
\hline 21 & Michiels, et al., $2019^{29}$ & $7,9(1,1)$ & $7,3(0,9)$ & 0,60 & $<0,0001$ & $7,7(0,8)$ & $7,6(1,0)$ & 0,10 & $<0,01$ & 0,50 & 0,067 \\
\hline 22 & Mourao, et al., $2012^{30}$ & $9,9(2,1)$ & 9,3 (NE) & 0,60 & 0,001 & $9,5(1,8)$ & 10,2 (NE) & $-0,70$ & 0,001 & 1,30 & 0,001 \\
\hline 23 & Sarayani, et al., $2018^{31}$ & $7,84(1,17)$ & $6,97(1,14)$ & 0,87 & $>0,05$ & $8,16(1,66)$ & $7,09(1,78)$ & 1,07 & $>0,05$ & $-0,20$ & $>0,05$ \\
\hline 24 & Scott, et al., $2006^{32}$ & 8,8 (NE) & 7,08 (NE) & 1,72 & 0,003 & 8,7 (NE) & 8,0 (NE) & 0,70 & $<0,05$ & 1,02 & $<0,05$ \\
\hline 25 & Siaw, et al., $2017^{33}$ & $8,5(1,5)$ & 8,1 (NE) & 0,40 & $<0,05$ & $8,6(1,4)$ & 8,5 (NE) & 0,10 & $<0,05$ & 0,30 & 0,014 \\
\hline 26 & Taveira, et al., 2010 & $8,1(1,5)$ & $7,2(1,6)$ & 0,90 & $<0,05$ & $7,9(1,1)$ & $7,9(1,5)$ & 0,00 & $>0,05$ & 0,90 & $<0,05$ \\
\hline 27 & Taveira, et al., $2011^{35}$ & $8,3(1,7)$ & $7,4(1,2)$ & 0,90 & $<0,05$ & $8,5(1,9)$ & $8,4(2,0)$ & 0,10 & $>0,05$ & 0,80 & $<0,05$ \\
\hline 28 & Wishah, et al., 2014 & $8,9(1,6)$ & $7,2(0,9)$ & 1,70 & 0,01 & $8,2(1,3)$ & $7,9(1,3)$ & 0,30 & 0,01 & 1,40 & $<0,05$ \\
\hline 29 & $\begin{array}{c}\text { Withidpanyawong, et } \\
\text { al., } 2018^{37}\end{array}$ & $9,21(1,84)$ & $7,84(1,96)$ & 1,37 & $<0,001$ & $9,08(1,47)$ & $8,87(1,81)$ & 0,21 & 0,270 & 1,16 & $<0,001$ \\
\hline 30 & Wu, et al., $2018^{38}$ & $8,16(\mathrm{NE})$ & $7,90(1,25)$ & 0,27 & $>0,05$ & $8,16(\mathrm{NE})$ & $8,02(1,23)$ & 0,14 & $>0,05$ & 0,13 & 0,30 \\
\hline
\end{tabular}


Respecto a la duración de cada cita y el intervalo de tiempo entre una y otra, esta información no fue especificada en todos los estudios, pero básicamente todos los estudios en los grupos de intervención implicaron entrevistas farmacéuticas como principal fuente de información y la realización de pruebas de laboratorio para la medición objetiva de resultados; la totalidad de los estudios trabajaron basados en protocolos de intervención para llevar a cabo la atención farmacéutica; nueve estudios incluyen en sus publicaciones una descripción detallada y estructurada de los programas de atención farmacéutica desarrollados ${ }^{11-13,16,18,23,32,34,35}$. En cuanto al tipo de sesiones llevadas a cabo por los grupos de intervención, 22 estudios trabajaron con citas individuales únicamente, 5 estudios con sesiones grupales ${ }^{16,34,35,37,38}$ y 3 estudios combinaron sesiones individuales y grupales ${ }^{12,31,32}$. Además 18 estudios realizaron sesiones presenciales, 1 estudio realizó únicamente sesiones vía telefónica ${ }^{26}$ y 11 estudios combinaron sesiones presenciales con seguimientos por vía telefóni$\mathrm{ca}^{9,14,15,17,21,22,31-33,36,37}$.

En cuanto a la provisión de la atención habitual, correspondiente al grupo control, en 10 estudios estuvo a cargo del médico; en 6 estudios, médico y enfermera; en otros 6 estudios, médico, enfermera y nutricionista; en 5 estudios el farmacéutico 11,18,25,28,29; en 2 estudios médico, enfermera y farmacéutico; y en 1 estudio la enfermera. Por otra parte, en 7 estudios les fue proporcionado un glucómetro ${ }^{12,21,22,25,28,31,32}$ a cada paciente, tanto del grupo control como del grupo de intervención, para la monitorización diaria de la glucosa.

\section{Hemoglobina glicosilada}

En el grupo de intervención, el valor medio de la HbA1c disminuyó desde el inicio hasta el final, en todos los estudios, pero alcanzó una disminución en la diferencia media neta estadísticamente significativa en 27 estudios: el rango osciló entre 0,4 \% y 3,3\%. Cabe destacar que en 14 estudios dicha disminución en la diferencia media neta fue estadísticamente significativa y superior o igual a $1 \%$. Por su parte, en el grupo control se alcanzó una disminución en la diferencia media neta estadísticamente significativa, únicamente en 8 estudios, y el rango osciló entre: $0,1 \%$ y $1 \%$, de los cuales solo 1 estudio fue igual a $1 \%$.

En cuanto a la disminución en la diferencia media neta mostrada en el cambio de $\mathrm{HbA} 1 \mathrm{c}$ desde el inicio hasta el final del seguimiento, entre el grupo de intervención y el grupo control, fue significativa en 22 es- tudios y el rango osciló entre: 0,3 y 2,3\%. Los valores de $\mathrm{HbA} 1 \mathrm{c}$ del grupo de intervención y del grupo control de los 30 ensayos controlados aleatorios incluidos en la presente revisión sistemática se detallan en la Tabla 3.

\section{Discusión}

Los dos principales tipos de intervenciones de atención farmacéutica que los farmacéuticos llevaron a cabo en los grupos de intervención son: 1) las dirigidas a la educación del paciente, en las que destacan la educación sobre la enfermedad y las complicaciones; la modificación en el estilo de vida, principalmente dieta y ejercicio, y también la modificación de comportamientos, particularmente el autocuidado y la adherencia a la medicación; 2) las dirigidas a operar cambios en la farmacoterapia del paciente, tanto las relacionadas con modificaciones en la estrategia farmacoterapéutica, como también en la dosificación (dosis y pauta); evidentemente este tipo de intervenciones deben realizarse en coordinación con el médico. Ambas estrategias son complementarias, por lo cual fueron implementadas de forma conjunta en la mayoría de los estudios. La diversidad en las intervenciones puede estar relacionada con los diferentes roles y la integración de los farmacéuticos dentro de los equipos multidisciplinarios de salud en los diferentes ámbitos asistenciales en cada país.

Los resultados del control glicémico detallados en la presente investigación concuerdan con los presentados por Pousinho et al. en sus dos revisiones sistemáticas: la primera publicada en 2016, denominada Intervenciones de los farmacéuticos en el tratamiento de la DM tipo 2: una revisión sistemática de ensayos controlados aleatorios, en la cual reporta que la disminución de la diferencia en el cambio de $\mathrm{HbA} 1 \mathrm{c}$ desde el inicio hasta el final del seguimiento, entre el grupo de intervención y el grupo control, osciló entre $0,18 \%$ y $2,1 \%$. Posteriormente, en su revisión sistemática de ensayos controlados aleatorios, publicada en 2020 y denominada Intervenciones del farmacéutico clínico en el manejo de la DM tipo 2, reporta una disminución de la diferencia en el cambio de $\mathrm{HbA1c}$ desde el inicio hasta el final del seguimiento entre el grupo de intervención y el grupo control que osciló entre 0,05 y 2,1\%39. En cuanto a la significación clínica de estos resultados se puede destacar que, según el Estudio Prospectivo de Diabetes del Reino Unido (UKPDS 35), por cada reducción del $1 \%$ en los niveles de HbA1c, disminuye el riesgo de muerte relacionada con la diabetes en un $21 \%$, el riesgo de in- 
farto de miocardio en un $14 \%$ y el riesgo de complicaciones microvasculares en un $37 \%$.

Las evidencias procedentes de los ensayos controlados aleatorios incluidos en la presente investigación indican que los farmacéuticos pueden contribuir positivamente en el manejo de los pacientes ambulatorios con DM tipo 2; este tipo de intervenciones de atención farmacéutica podrían tener mayor alcance si fueran parte de la atención habitual ofrecida a los pacientes.

\section{Limitaciones}

La presente revisión sistemática cuenta con algunas limitaciones. En primer lugar, a pesar de que los ensayos controlados aleatorios tienen un diseño de estudio robusto, la mayoría de los estudios incluidos presentaron debilidades metodológicas al ser evaluados con la herramienta de riesgo de sesgo de Cochrane, debido principalmente a la insuficiente información al respecto publicada en los artículos. En segundo lugar, únicamente se consideró el cambio de los valores medios de hemoglobina glicosilada, sin incluir el cambio en la presión arterial, el perfil lipídico y el índice de masa corporal, que también fueron reportados en la mayoría de los estudios. Además, la revisión sistemática fue realizada por un solo autor y el protocolo no fue registrado.

\section{Conclusión}

Debido al rol infravalorado que tiene el farmacéutico en el contexto de las intervenciones clínicas, en los diferentes ámbitos en los que se desempeña, ya sea en la farmacia comunitaria, clínica u hospitalaria, esta revisión sistemática recopila evidencias que demuestran el efecto que tiene el farmacéutico en la mejora del control glicémico de pacientes ambulatorios con DM tipo 2 mediante la implementación de intervenciones de atención farmacéutica que no se limitan únicamente a aspectos educativos sino que inciden en cambios en la farmacoterapia del paciente, estableciendo metas terapéuticas individuales y desarrollando planes terapéuticos integrales que generan resultados clínicos en los pacientes y que impactan los sistemas de salud. Por otra parte, estos hallazgos apoyan la idea de considerar al farmacéutico como un elemento fundamental en los equipos multidisciplinarios de salud en la atención a pacientes con DM y a su vez promueven la implementación de este enfoque en los sistemas de salud de todo el mundo, donde los farmacéuticos no tienen una participación activa en el tratamiento de este tipo de pacientes.

\section{Financiamiento}

La investigación fue financiada por fondos propios del autor.

\section{Referencias bibliográficas}

1. Organización Mundial de la Salud. Informe Mundial sobre la Diabetes. Geneva OMS.

2016. 88p. Fecha de consulta: 25 de febrero de 2021. Disponible en: https://www.who. int/publications/i/item/9789241565257

2. Benedict AW, Spence MM, Sie JL, Chin HA, Ngo CD, Salmingo JF, et al. Evaluation of a Pharmacist-Managed Diabetes Program in a Primary Care Setting Within an Integrated Health Care System. JMCP. 2018;24(2):11422. DOI: 10.18553/jmcp.2018.24.2.114

3. Abdulrhim SH, Saleh RA, Mohamed Hussain MA, Al Raey H, Babiker AH, Kheir N, et al. Impact of a collaborative pharmaceutical care service among patients with diabetes in an ambulatory care setting in Qatar: A multiple time series study. Value Health Reg Issues. 2019;19:45-50. DOI: 10.1016/j. vhri.2018.12.002

4. Lai YR, Huang CC, Chiu WC, Liu RT, Tsai $\mathrm{NW}$, Wang $\mathrm{HC}$, et al. HbA1C Variability is strongly associated with the severity of cardiovascular autonomic neuropathy in patients with type 2 diabetes after longer diabetes duration. Front Neurosci. 2019;13:458. DOI: 10.3389/fnins.2019.00458

5. American Diabetes Association. 1. Improving Care and Promoting Health in Populations: Standards of Medical Care in Diabetes-2020. Diabetes Care. 2020;43(Supplement 1): S7S13. DOI: $10.2337 /$ dc20-S001

6. Pousinho S, Morgado M, Falcão A, Alves G. Pharmacist interventions in the management of type 2 diabetes mellitus: A systematic review of randomized controlled trials. JMCP. 2016;22(5):493-515. DOI: 10.18553/jmcp.2016.22.5.493

7. Liberati A, Altman DG, Tetzlaff J, Mulrow C, Gotzsche PC, loannidis JPA, et al. The PRISMA statement for reporting systematic reviews and meta-analyses of studies that evaluate healthcare interventions: explanation and elaboration. BMJ. 2009;339:b2700. DOI: 10.1136/bmj.b2700

8. Manual Cochrane de revisiones sistemáticas de intervenciones, versión 5.1.0 Centro Cochrane Iberoamericano. Barcelona, España. 2021. Disponible en: https:// es.cochrane.org/sites/es.cochrane.org/files/ public/uploads/Manual Cochrane 510 reduit.pdf 
9. Aguiar PM, Silva CH, Chiann C, Lima Dórea E, Lyra DP, Storpirtis S. Pharmacist-physician collaborative care model for patients with uncontrolled type 2 diabetes in Brazil: results from a randomized controlled trial. J Eval Clin Pract. 2018;24(1):22-30. DOI: 10.1111/ jep.12606

10. Ali M, Schifano F, Robinson P, Phillips G, Doherty L, Melnick P, et al. Impact of community pharmacy diabetes monitoring and education programme on diabetes management: a randomized controlled study. Diabet Med. 2012;29(9):e326-e333. DOI: 10.1111/j.1464-5491.2012.03725.x

11. Ayadurai S, Sunderland VB, Tee LBG, MD Said SN, Hattingh HL. Structured tool to improve clinical outcomes of type 2 diabetes mellitus patients: A randomized controlled trial. J Diabetes. 2018;10(12):96576. DOl: 10.1111/1753-0407.12799

12. Castejón AM, Calderón JL, Pérez A, Millar C, McLaughlin-Middlekauff J, Sangasubana $\mathrm{N}$, et al. A community-based pilot study of a diabetes pharmacist intervention in Latinos: impact on weight and hemoglobin A1c. J Health Care Poor Underserved. 2014;24(4A):48-60. DOI: 10.1353/ hpu.2014.0003

13. Chan C-W, Siu S-C, Wong CKW, Lee VWY. A pharmacist care program: positive impact on cardiac risk in patients with type 2 diabetes. J Cardiovasc Pharmacol Ther. 2012;17(1):57-64. DOI: 10.1177/1074248410396216

14. Chen J-H, Ou H-T, Lin T-C, Lai EC-C, Yang Kao Y-H. Pharmaceutical care of elderly patients with poorly controlled type 2 diabetes mellitus: a randomized controlled trial. Int J Clin Pharm. 2016;38(1):88-95. DOI: 10.1007/ s11096-015-0210-4

15. Clifford RM, Davis WA, Batty KT, Davis TME. Effect of a pharmaceutical care program on vascular risk factors in type 2 diabetes: The Fremantle Diabetes Study. Diabetes Care. 2005;28(4):771-6. DOI: 10.2337/ diacare.28.4.771

16. Cohen LB, Taveira TH, Khatana SAM, Dooley AG, Pirraglia PA, Wu W-C. Pharmacistled shared medical appointments for multiple cardiovascular risk reduction in patients with type 2 diabetes. Diabetes Educ. 2011;37(6):801-12. DOI: 10.1177/0145721711423980

17. Farsaei S, Sabzghabaee AM, Zargarzadeh AH, Amini M. Effect of pharmacist-led patient education on glycemic control of type 2 diabetics: a randomized controlled trial. J Res Med Sci. 2011;16(1):43-9. Disponible en: https://www.ncbi.n/m.nih.gov/pmc/articles/ PMC3063424/

18. Fornos JA, Andrés NF, Andrés JC, Guerra MM, Egea B. A Pharmacotherapy follow-up program in patients with type-2 diabetes in community pharmacies in Spain. Pharm World Sci. 2006;28(2):65-72. DOI: 10.1007/ s11096-006-9003-0

19. Jaber $L A$, Halapy $H$, Fernet $M$, Tummalapalli S, Diwakaran $\mathrm{H}$. Evaluation of a pharmaceutical care model on diabetes management. Ann Pharmacother. 1996;30(3):238-43. DOI: 10.1177/106002809603000305

20. Jacobs M, Sherry PS, Taylor LM, Amato M, Tataronis GR, Cushing G. Pharmacist Assisted Medication Program Enhancing the Regulation of Diabetes (PAMPERED) study. J Am Pharm Assoc. 2012;52(5):613-21. DOI: 10.1331/JAPhA.2012.10183

21. Jahangard-Rafsanjani Z, Sarayani A, Nosrati M, Saadat N, Rashidian A, Hadjibabaie $M$, et al. Effect of a community pharmacist-delivered diabetes support program for patients receiving specialty medical care: a randomized controlled trial. Diabetes Educ. 2015;41(1):127-35. DOI: 10.1177/0145721714559132

22. Jarab AS, Alqudah SG, Mukattash TL, Shattat G, Al-Qirim T. Randomized controlled trial of clinical pharmacy management of patients with type 2 diabetes in an outpatient diabetes clinic in Jordan. JMCP. 2012;18(7):516-26. DOI: 10.18553/ jmcp.2012.18.7.516

23. Javaid Z, Imtiaz U, Khalid I, Saeed H, Khan $R Q$, Islam $M$, et al. A randomized control trial of primary care-based management of type 2 diabetes by a pharmacist in Pakistan. BMC Health Serv Res. 2019;19(1):409. DOI: 10.1186/s12913-019-4274-z

24. Korcegez El, Sancar M, Demirkan K. Effect of a Pharmacist-Led Program on Improving Outcomes in Patients with Type 2 Diabetes Mellitus from Northern Cyprus: A Randomized Controlled Trial. J Manag Care Spec Pharm. 2017;23(5):573-82. DOI: 10.18553/jmcp.2017.23.5.573

25. Krass I, Armour CL, Mitchell B, Brillant M, Dienaar R, Hughes J, et al. The Pharmacy Diabetes Care Program: assessment of a community pharmacy diabetes service model in Australia. Diabet Med. 2007;24(6):677-83. DOI: 10.1111/j.14645491.2007.02143.x

26. Lauffenburger JC, Ghazinouri R, Jan S, Makanji S, Ferro CA, Lewey J, et al. Impact of a novel pharmacist-delivered behavioral intervention for patients with poorlycontrolled diabetes: The ENhancing outcomes through Goal Assessment and Generating Engagement in Diabetes Mellitus (ENGAGE-DM) pragmatic randomized trial. PLoS One. 2019;14(4):e0214754. DOI: 10.1371/journal.pone.0214754

27. Mazroui NR, Kamal MM, Ghabash NM, Yacout TA, Kole PL, McElnay JC. Influence of pharmaceutical care on health outcomes 
in patients with Type 2 diabetes mellitus. Br J Clin Pharmacol. 2009;67(5):547-57. DOI: 10.1111/j.1365-2125.2009.03391.x

28. Mehuys E, Van Bortel L, De Bolle L, Van Tongelen I, Annemans L, Remon J-P, et al. Effectiveness of a community pharmacist intervention in diabetes care: a randomized controlled trial. J Clin Pharm Ther. 2011;36(5):602-13. DOl: 10.1111/j.13652710.2010.01218.x

29. Michiels Y, Bugnon O, Chicoye A, Dejager S, Moisan C, Allaert F-A, et al. Impact of a Community Pharmacist-Delivered Information Program on the Follow-up of Type-2 Diabetic Patients: A Cluster Randomized Controlled Study. Adv Ther. 2019;36(6):1291-1303. DOI: 10.1007/s12325019-00957-y

30. Mourao AOM, Ferreira WR, Martins MAP, Reis AMM, Carrillo MRG, Guimaraes $A G$, et al. Pharmaceutical care program for type 2 diabetes patients in Brazil: a randomised controlled trial. Int J Clin Pharm. 2013;35(1):79-86. DOI: 10.1007/s11096-0129710-7

31. Sarayani A, Mashayekhi M, Nosrati M, Jahangard-Rafsanjani Z, Javadi M, Saadat $N$, et al. Efficacy of a telephone-based intervention among patients with type-2 diabetes; a randomized controlled trial in pharmacy practice. Int J Clin Pharm. 2018;40(2):345-53. DOI: 10.1007/s11096-0180593-0

32. Scott DM, Boyd ST, Stephan M, Augustine SC, Reardon TP. Outcomes of pharmacistmanaged diabetes care services in a community health center. Am J Health Syst Pharm. 2006;63(21):2116-22. DOI: 10.2146/ ajhp060040

33. Siaw MYL, Malone DC, Ko Y, Lee JY-C. Cost-effectiveness of multidisciplinary collaborative care versus usual care in the management of high-risk patients with diabetes in Singapore: Short-term results from a randomized controlled trial. J Clin Pharm Ther. 2018;43(6):775-83. DOI: 10.1111/ jcpt.12700
34. Taveira TH, Friedmann PD, Cohen LB, Dooley AG, Khatana SAM, Pirraglia PA, et al. Pharmacist-Led Group Medical Appointment Model in Type 2 Diabetes. Diabetes Educ. 2010;36(1):109-17. DOI: 10.1177/0145721709352383

35. Taveira TH, Dooley AG, Cohen LB, Khatana SAM, Wu W-C. Pharmacist-Led Group Medical Appointments for the Management of Type 2 Diabetes with Comorbid Depression in Older Adults. Ann Pharmacother. 2011;45(11):1346-55. DOI: 10.1345/aph.1Q212

36. Wishah RA, Al-Khawaldeh OA, Albsoul AM. Impact of pharmaceutical care interventions on glycemic control and other healthrelated clinical outcomes in patients with type 2 diabetes: Randomized controlled trial. Diabetes Metab Syndr. 2015;9(4):271-6. DOI: 10.1016/j.dsx.2014.09.001

37. Withidpanyawong U, Lerkiatbundit S, Saengcharoen W. Family-based intervention by pharmacists for type 2 diabetes: A randomised controlled trial. Patient Educ Couns. 2019;102(1):85-92. DOI: 10.1016/j. pec.2018.08.015

38. Wu W-C, Taveira TH, Jeffery S, Jiang L, Tokuda $L$, Musial J, et al. Costs and effectiveness of pharmacist-led group medical visits for type-2 diabetes: A multi-center randomized controlled trial. PLoS ONE. 2018;13(4):e0195898. DOI: 10.1371/journal. pone.0195898

39. Pousinho S, Morgado M, Plácido Al, Roque F, Falcão A, Alves G. Clinical pharmacists' interventions in the management of type 2 diabetes mellitus: a systematic review. Pharm Pract (Granada). 2020;18(3):2000. DOI: 10.18549/PharmPract.2020.3.2000

40. Stratton IM, Adler Al, W Neil HA, Matthews DR, Manley SE, Cull CA, et al. Association of glycaemia with macrovascular and microvascular complications of type 2 diabetes (UKPDS 35): prospective observational study. BMJ. 2000;321(7258):405-12. DOI: 10.1136/ bmj.321.7258.405 